\title{
Some Properties of Secretory IgA Purified from Bovine Colostrum
}

\author{
Yoshihiro Kanamaru, Yasuo Kuzuya and Tamotsu Tanahashi \\ Department of Poultry and Animal Sciences, Faculty of Agriculture, \\ Gifu University, Kakamigahara-shi, Gifu 504, Japan \\ Received October 29, 1981
}

\begin{abstract}
The major component of a purified sample of secretory IgA (SIgA) in colostrum was revealed as a single peak on gel filtration with Sepharose $6 \mathrm{~B}$, having an estimated molecular weight of 540,000 . The existence of a higher molecular weight component was suggested by a small shoulder on the ascending limb of the peak, but another component of IgA reported as IgA lacking the secretory component (SC) could not be found. When the purified SIgA was concentrated by dialysis against polyethylene glycol, its molecular size was apparently significantly decreased.

Analysis by polyacrylamide gel electrophoresis in the presence of sodium dodecyl sulfate (SDSPAGE) showed that all SC in SIgA binds covalently. The band corresponding to the J chain was easily detected when a reduced and alkylated sample was analysed. Estimation of the molecular weight by SDS-PAGE gave the following values for each of the constituent polypeptide chains of bovine colostral SIgA: SC, 76,000; H chain, 62,000; L chain, 23,000; and J chain, 18,000. The molecular weight of the whole molecule was calculated to be 434,000 .

Analysis of carbohydrates by gas-liquid chromatography showed $6.8 \%$ neutral and amino hexoses, consisting of $0.4 \%$ fucose, $1.8 \%$ mannose, $1.1 \%$ galactose and $3.5 \%$ glucosamine. Galactosamine, which has been found in bovine free secretory component from milk, could not be detected.
\end{abstract}

Human secretory immunoglobulin A (SIgA) is composed of four different kinds of polypeptide chains: $\mathrm{H}$ and $\mathrm{L}$ chains, a $\mathrm{J}$ chain and a secretory component (SC). ${ }^{1,2}$ The complete structural formula is expressed as $\left(\mathrm{H}_{2} \mathrm{~L}_{2}\right)_{2}$. $\mathrm{J} \cdot \mathrm{SC}$. This may also be the case for bovine SIgA, but only a few data are available with respect to its molecular structure. Heterogeneity was reported for bovine SIgA in its molecular size and in the content of SC. Purified bovine nasal SIgA was found to exist in two polymeric forms (15.3 and $12.2 \mathrm{~S}){ }^{3)}$ The 15.3 $\mathrm{S}$ IgA dissociated into $12.2 \mathrm{~S}$ and $7 \mathrm{~S}$ components upon treatment with $4 \mathrm{M}$ guanidine- $\mathrm{HCl}$. Similar hydrophobic polymers of SIgA were found in bovine colostrum and other secretions, being most pronounced in tears. ${ }^{4}$ Recently IgA lacking SC was found in a preparation purified from bovine bronchioalveolar washings, although most of the IgA contained SC. ${ }^{5)}$ The presence of IgA with and without SC was also reported previously for a preparation of IgA from colostrum. ${ }^{4}$

As described in the preceding paper, ${ }^{27)}$ the authors prepared a highly purified bovine colostral SIgA. The purpose of the study reported here were to analyse its molecular size, to detect its constituent polypeptides and to determine its carbohydrate content.

\section{MATERIALS AND METHODS}

Proteins. Bovine colostral SIgA and free secretory component (FSC) were prepared as described in the preceding paper. ${ }^{27)}$ The samples were lyophilized after exhaustive dialysis against distilled water. Some SIgA samples from an anti-IgG-Fc-Sepharose column ${ }^{27)}$ were concentrated by dialysis against polyethylene glycol ( $P E G{ }^{\sharp} 20,000$, Nakarai Chemicals, Ltd., Kyoto). Cellulose tubing (Visking Company, Chicago, U.S.A.) containing $c a .50 \mathrm{ml}$ of the purified SIgA solution was put on solid polyethylene glycol at $4{ }^{\circ} \mathrm{C}$. When the $\mathrm{SIgA}$ solution was concentrated (below $5 \mathrm{ml}$ ), the cellulose tubing was washed exhaustively with running tap water and the SIgA solution was dialysed against the buffer used for gel filtration at $4{ }^{\circ} \mathrm{C}$. The solution was then removed from the tubing and stored at 
$4^{\circ} \mathrm{C}$. Bovine IgGl was prepared from colostrum as described previously. ${ }^{6}$

Gel filtration. Gel filtration was performed on a column $(2.5 \times 96 \mathrm{~cm})$ of Sepharose 6B (Pharmacia Fine Chemicals, Uppsala, Sweden) equilibrated with $0.05 \mathrm{M}$ Tris- $\mathrm{HCl}$ buffer, $\mathrm{pH} 8.0$, containing $0.15 \mathrm{M} \mathrm{NaCl}, 1 \mathrm{~mm}$ EDTA and $0.02 \% \mathrm{NaN}_{3}$. The standard proteins used for the molecular weight estimation were purchased from Pharmacia Fine Chemicals, Uppsala, Sweden.

Polyacrylamide gel electrophoresis in the presence of sodium dodecyl sulfate (SDS-PAGE). SDS-PAGE was carried out essentially according to the method of Weber and Osborn. ${ }^{7)}$ Unreduced samples were electrophoresed in $5 \%$ gels. Other samples were electrophoresed in $10 \%$ gels with 2-mercaptoethanol (Wako Pure Chemical Industries, Ltd., Osaka). Protein and carbohydrate were localized in gels using Coomassie Brilliant Blue R-250 (Nakarai Chemicals, Ltd., Kyoto) and periodic acid-Schiff (PAS) reagent ${ }^{8)}$ respectively. The standard proteins used for the molecular weight estimation were obtained from Sigma Chemical Company (St. Louis, U.S.A.).

Disc electrophoresis in $8 \mathrm{M}$ urea. Disc electrophoresis was carried out at $\mathrm{pH} 4.3$ in $5.6 \%$ gels in the presence of $8 \mathrm{M}$ urea. ${ }^{9)}$ Protein was visualized with Amido Black 10B (Wako Pure Chemical Industries, Ltd., Osaka).

Reduction and alkylation ${ }^{3)} \mathrm{SIgA}$ was reduced in $0.2 \mathrm{M}$ Tris- $\mathrm{HCl}$ buffer, $\mathrm{pH} 8.0$, containing $5 \mathrm{M}$ urea, using $10 \mathrm{~mm}$ dithiothreitol (Nakarai Chemicals, Ltd., Kyoto) at room temperature for $2 \mathrm{hr}$. Alkylation was done with $40 \mathrm{~mm}$ iodoacetamide (Tokyo Kasei Kogyo Co., Ltd., Tokyo).

Determination of protein concentration. The concentration of SIgA was determined spectrophotometrically using an extinction coefficient of $E_{280}^{1 \%}=13.9 .^{10}$

Carbohydrate analysis. Neutral and amino hexoses were determined by gas-liquid chromatography of alditol acetates according to the method of Tomana $e$ al $^{11)}$ with a slight modification. A sample of a known concentration in $0.01 \mathrm{~N} \mathrm{HCl}$ was digested overnight at $37^{\circ} \mathrm{C}$ with pepsin (Sigma Chemical Company, St. Louis, U.S.A.), at an enzyme to substrate ratio of $1: 200$. It was then hydrolyzed in $1 \mathrm{~N} \mathrm{HCl}$ for 2,4 and $8 \mathrm{hr}$ at $100^{\circ} \mathrm{C}$. These hydrolysates were used for the following determinations: the $2 \mathrm{hr}$ one for fucose, the $4 \mathrm{hr}$ one for mannose and the $8 \mathrm{hr}$ one for galactose and aminohexoses. Carbohydrates were converted to their alditol acetates by the procedure of Niedermeier. ${ }^{12)}$ Alditol acetates in $\mathrm{CH}_{2} \mathrm{Cl}_{2}$ (Nakarai Chemicals, Ltd., Kyoto) were analysed with a Shimadzu GC5 gas chromatograph equipped with dual-flame ionization detectors. The neutral sugars were chromatographed on a glass column $(0.3 \times 200 \mathrm{~cm})$ packed with $0.2 \%$ polyethylene glycol succinate, $0.2 \%$ polyethylene glycol adipate and $0.4 \%$ silicone XF-1150 on $100 / 120$ mesh Gas-Chrom $P$ (purchased from Wako Pure Chemical Industries, Ltd., Osaka). The starting temperature of $140^{\circ} \mathrm{C}$ was programmed to $200^{\circ} \mathrm{C}$ at the rate of $5^{\circ} \mathrm{C} / \mathrm{min}$. Arabinose was used as the internal standard. The amino sugars were chromatographed on a glass column $(0.3 \times 200 \mathrm{~cm})$ packed with $3 \%$ Poly-A 103 on $100 / 200$ mesh Gas-Chrom Q (purchased from Wako Pure Chemical Industries, Ltd., Osaka) using D-mannosamine as the internal standard. The starting temperature of $210^{\circ} \mathrm{C}$ was programmed to $260^{\circ} \mathrm{C}$ at the rate of $5^{\circ} \mathrm{C} / \mathrm{min}$.

\section{RESULTS AND DISCUSSION}

\section{Gel filtration studies}

It has been reported ${ }^{4)}$ that recycling gel filtration studies of the SIgA-rich fraction of bovine colostral whey showed the appearance of two IgA-rich peaks, of estimated molecular weights of 440,000 and 350,000 . The concentration of IgA was nearly equal in both fractions. The larger component reacted with both the anti- $\alpha$ chain (the heavy chain of IgA) and the anti-free secretory component (FSC) antisera, whereas the smaller component did not react with the anti-FSC antiserum, suggesting that both IgA with and without SC exists in equal amounts in bovine colostrum. These observations were made, however, using an IgA fraction containing large amounts of IgGl. Similar IgA lacking SC was found in a purified $\operatorname{IgA}$ preparation from bovine bronchio-alveolar washings. ${ }^{5}$

In the present study, in order to evaluate the size heterogeneity of bovine colostral SIgA, the purified sample was first examined by gel filtration. Figure 1 shows its elution profile on Sepharose 6B. Only one peak was observed with a very small shoulder on the ascending limb of the peak (represented by open circles). The molecular weight of the component in the peak was estimated to be 540,000 , as shown in the inset of Fig. 1, and no smaller components were found, suggesting that the IgA sample we purified contains no IgA lacking SC. It is possible that IgA lacking SC had already been removed before the purification of $\operatorname{IgA}$ by affinity chromatography on anti-IgG-FcSepharose. ${ }^{27)}$ However, it seems likely that the amount of $\mathrm{IgA}$ lacking SC, if present, is much 


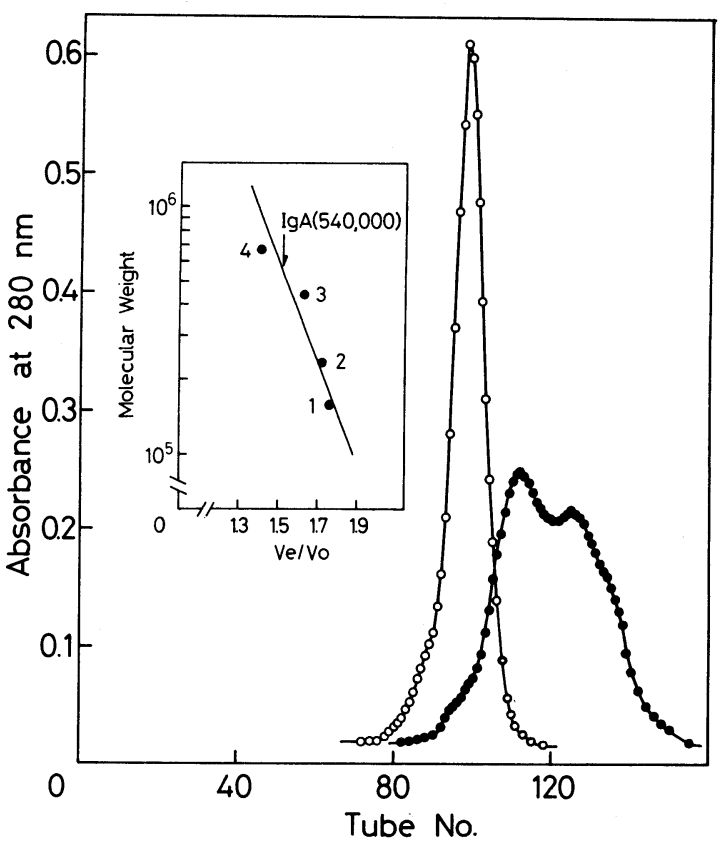

FIG. 1. Gel Filtration Profile of Purified Bovine Colostral IgA.

The column $(2.5 \times 96 \mathrm{~cm})$ was equilibrated with $0.05 \mathrm{M}$ Tris- $\mathrm{HCl}$ buffer, $\mathrm{pH} 8.0$, containing $0.15 \mathrm{M} \mathrm{NaCl}, 1 \mathrm{~mm}$ EDTA and $0.02 \% \mathrm{NaN}_{3}$. The flow rate was $20 \mathrm{ml} / \mathrm{hr}$ and the eluate was collected in $3 \mathrm{ml}$ fractions. The experiment was done at room temperature. Open circles represent the elution profile of the lyophilized IgA sample and filled circles represent the elution profile of the concentrated sample with polyethylene glycol. The inset shows the molecular weight estimation of bovine colostral IgA using the following standard proteins: 1 , aldolase $(158,000) ; 2$, catalase $(232,000) ; 3$, ferritin $(440,000) ; 4$, thyroglobulin $(669,000)$.

smaller than that of IgA with SC, since a) there is a large amount of $\mathrm{SC}$ in the free form in bovine colostrum ${ }^{13)}$ and b) all SC in the SIgA molecule from bovine colostrum was found to be bound covalently with IgA, as shown in the electrophoresis section.

An aggregated form of bovine SIgA was reported in several studies. ${ }^{3 \sim 5,19)}$ A considerable amount of SIgA was found to exist in the $15.3 \mathrm{~S}$ polymer in bovine nasal secretions $\mathrm{s}^{3)}$ and was suggested to be a trimer of IgA. The existence of hydrophobic polymers of IgA in colostrum and other external secretions was also suggested ${ }^{4}{ }^{4}$ The small shoulder found on gel filtration (Fig. 1) may therefore represent similar aggregated IgA, but definite identification could not be made due to shortage of the sample.

A quite different elution profile was observed when the purified SIgA sample was concentrated by dialysis against polyethylene gly- col and then applied to the Sepharose 6B column. As shown in Fig. 1 (represented by filled circles), SIgA was eluted apparently in two broad peaks, the positions of which correspond to much smaller molecular weights than that of the lyophilized SIgA sample. On analysis of the components in both peaks by SDS-PAGE (results not shown), quite similar patterns were observed. Unreduced samples gave only one stained band that migrated considerably faster than the lyophilized SIgA. These results suggest that the molecular size of bovine SIgA was significantly decreased by the treatment with polyethylene glycol. These observations cannot immediately be explained. SIgA may be in a more compact form due to the possible interaction with some polyethylene glycols, which can penetrate the dialysis membrane. When bovine IgG1 or IgG2 was examined similarly, no differences were found between the concentrated samples using 
polyethylene glycol and the lyophilized samples on both gel filtration and SDS-PAGE. Further studies should be done in order to explain the unusual decrease in the apparent molecular size of bovine colostral SIgA.

\section{Electrophoretic studies}

The purified bovine SIgA in the peak in Fig. 1 was analysed by SDS-PAGE without reducing agents. As shown in Fig. 2A, only a single protein band was observed near the top of the gel, suggesting that only one component was contained in the sample. In contrast with this, bovine IgG1 gave five minor protein bands in an experiment performed under the same conditions, which correspond to $\mathrm{H}_{2} \mathrm{~L}, \mathrm{H}_{2}, \mathrm{HL}$, $\mathrm{H}$ and $\mathrm{L}$. These results suggest that some interchain disulfide bonds of bovine IgG1 were reduced merely by boiling in $1 \%$ SDS. A similar sensitivity of interchain disulfide bonds was also reported for human $\operatorname{IgG}{ }^{14)}$ On the other hand, bovine colostral SIgA was shown to be resistant to the same treatment.

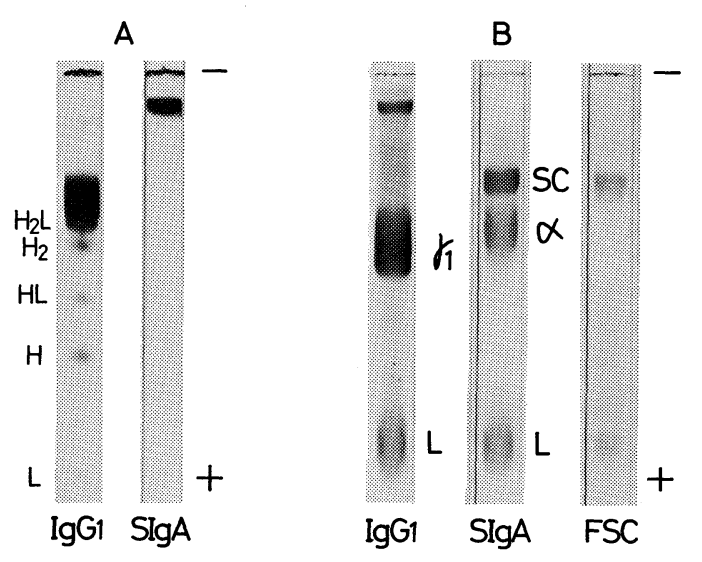

Fig. 2. SDS-PAGE Patterns of Bovine Colostral IgA, IgGl and FSC.

The samples were boiled with $1 \%$ SDS in the absence (A) and presence (B) of $1 \%$ 2-mercaptoethanol before electrophoresis. The gel concentrations were $5 \%$ (A) and $10 \%$ (B). Electrophoresis was carried out for $4 \mathrm{hr}$ (A) or $6 \mathrm{hr}$ (B) at a current of $7 \mathrm{~mA} /$ tube.

$\mathrm{L}$, light chain; $\mathrm{H}$, heavy chain; $\mathrm{HL}$, the molecule consisting of one $\mathrm{H}$ and one $\mathrm{L} ; \mathrm{H}_{2}$, the molecule consisting of two $\mathrm{H}$; $\mathrm{H}_{2} \mathrm{~L}$, the molecule consisting of two $\mathrm{H}$ and one $\mathrm{L} ; \gamma_{1}, \mathrm{H}$ of IgGl; $\alpha, \mathrm{H}$ of IgA; SC, secretory component.
When the SIgA sample was boiled with $1 \%$ 2 -mereaptoethanol in $1 \%$ SDS prior to electrophoresis, three constituent subunits were detected in a $10 \%$ gel (Fig. 2B). The band from the IgA sample with the slowest mobility was detected at a position identical with that of FSC isolated from colostrum. The band corresponding to the $\alpha$ chain (heavy chain of $\operatorname{IgA}$ ) migrated slightly slower than the $\gamma_{1}$ chain (heavy chain of IgG1). The band with the fastest mobility corresponds to the L chain, as compared to the gel of reduced IgG1.

The majority of SC, i.e., more than $80 \%$, appears to be covalently bound to $\alpha$ chain in human SIgA. ${ }^{17)}$ On the other hand, a substantial amount of SC in rabbit SIgA is noncovalently bound and can be dissociated by treatment with SDS followed by SDSPAGE. ${ }^{15,16)}$ The results of this study, which showed that no SC was detected unless the SIgA sample was boiled with $1 \%$ 2-mercaptoethanol in $1 \%$ SDS on SDS-PAGE (Figs. 2A and $2 \mathrm{~B}$ ), suggest that all $\mathrm{SC}$ bound to bovine colostral SIgA is disulfide linked.

In human $\operatorname{IgA}$, a subclass $\left(\operatorname{IgA} 2, \operatorname{Am}(2)^{+}\right)$ was found in which $H$ and $L$ chains are associated noncovalently. ${ }^{18)}$ Since no $\mathrm{L}$ chain band was detected in the $5 \%$ gel of our sample in the absence of reducing agents (Fig. 2A), the possibility of the presence of such a subclass can be ruled out, at least in bovine colostral SIgA.

The other polypeptide chain, called the J chain, has been found in secretory $\operatorname{IgA}$ of many species. ${ }^{20 \sim 22)}$ The $\mathbf{J}$ chain is present only in polymeric $\operatorname{IgA}$ and $\operatorname{IgM}$ and not in their monomers. In order to detect the counterpart in bovine SIgA, reduction in the presence of $5 \mathrm{M}$ urea followed by alkylation was carried out. Disc electrophoresis of the reduced and alkylated sample in $8 \mathrm{M}$ urea (Fig. 3) yielded a unique protein band, which migrated rapidly to the cathode. This band was not found in the reduced and alkylated IgG1 or FSC. The electrophoretic patterns of the reduced and alkylated bovine SIgA were similar to those reported for the $\mathbf{J}$ chain in human $\operatorname{SIgA},{ }^{20}$ ) suggesting the presence of a similar poly- 


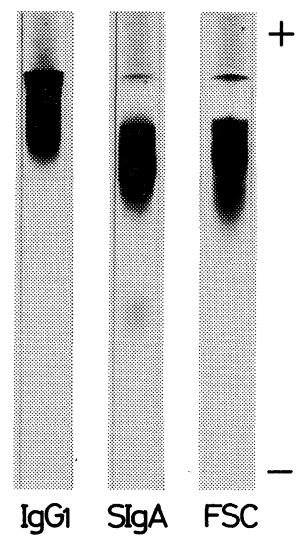

FIG. 3. Disc Electrophoresis of Reduced and Alkylated SIgA, IgGl and FSC in the Presence of $8 \mathrm{~m}$ Urea at Acid $\mathrm{pH}$.

Electrophoresis was carried out for $3 \mathrm{hr}$ at a current of $5 \mathrm{~mA} /$ tube.

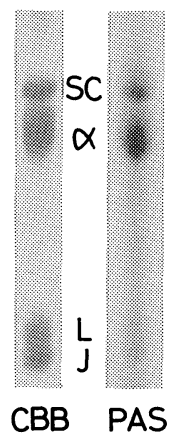

FIG. 4. SDS-PAGE Patterns of the Reduced and Alkylated Bovine Colostral SIgA.

The gel concentration was $10 \%$. Electrophoresis was carried out for $6 \mathrm{hr}$ a current of $7 \mathrm{~mA} /$ tube. After electrophoresis, the gels were stained with Coomassie Brilliant Blue (CBB) and periodic acid-Schiff (PAS) reagent.

$\mathrm{SC}$, secretory component; $\alpha$, heavy chain of $\operatorname{IgA}$; L, light chain; J, J chain.

peptide also in bovine SIgA. When the same sample was analysed by SDS-PAGE, a fourth protein band was found, migrating faster than the L chain band (Fig. 4). It also was stained by PAS reagent, suggesting that it represents bovine $\mathrm{J}$ chain. It is not clear why the band corresponding to the $\mathrm{J}$ chain could not be detected on SDS-PAGE when bovine colostral SIgA was merely boiled in the presence of $1 \%$ SDS and 1\% 2-mercaptoethanol. Butler et al. ${ }^{5}$ presented data showing that their SIgA sample

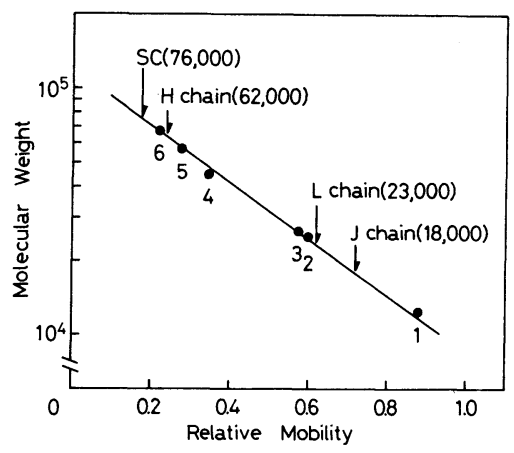

FIG. 5. Molecular Weight Estimation of Each Polypeptide of Bovine Colostral SIgA by SDS-PAGE.

The following standard proteins were used: 1 , cytochrome $c(13,000)$; 2 , L chain of IgGl $(25,000) ; 3, \alpha$-chymotrypsinogen A $(26,000) ; 4$, ovalbumin $(45,000) ; 5, \mathrm{H}$ chain of IgGl $(56,000) ; 6$, bovine serum albumin $(65,000)$.

purified from bovine bronchio-alveolar washings gave a band corresponding to the $\mathrm{J}$ chain on SDS-PAGE performed under almost the same experimental conditions. But in the same study the band was not seen when a slab gel was used. Therefore, the appearance of the band in SDS-gels may be influenced by subtle differences in the experimental conditions, e.g. amount of the sample loaded. Reduction followed by alkylation does not seem to be essential for detection of the band.

The molecular weights of the constituent subunits of bovine colostral SIgA were estimated by SDS-PAGE (Fig. 5), and the following results were obtained: $\mathrm{SC}, 76,000 ; \mathrm{H}$ chain, 62,000; L chain, 23,000; and $\mathrm{J}$ chain, 18,000 . All these values are consistent with the data reported previously., ${ }^{3,43}$ Based on the structural formula, $\left(\mathrm{H}_{2} \mathrm{~L}_{2}\right)_{2} \cdot \mathrm{J} \cdot \mathrm{SC}$, of human SIgA, the molecular weight of the whole molecule of bovine SIgA was calculated to be 434,000 , which is in good agreement with the analytical ultracentrifugation data of bovine nasal SIgA.

The molecular weight of 540,000 estimated from the data of gel filtration (Fig. 1) is much larger than that calculated from the data of SDS-PAGE (Fig. 5). The reason for this discrepancy may be that bovine colostral SIgA in the native state (i.e. in the buffer used for gel filtration) has a shape whose frictional ratio is 
TABle I. CARBohydrate Composition of Secretory Immunoglobulin A

Results are expressed as $\mathrm{g} / 100 \mathrm{~g}$ protein $(\mathrm{mols} / \mathrm{mol}$ protein). The molecular weight for bovine SIgA used for the calculation of molar composition was 434,000 .

\begin{tabular}{|c|c|c|}
\hline & $\begin{array}{c}\text { Bovine } \\
\text { SIgA }\end{array}$ & $\begin{array}{l}\text { Human } \\
\operatorname{SIgA}^{11)}\end{array}$ \\
\hline Fuc & $0.39 \quad(10.3)$ & $0.74 \quad(18.7)$ \\
\hline Man & $1.77 \quad(42.7)$ & $2.60 \quad(59.9)$ \\
\hline Gal & $1.09 \quad(26.3)$ & 1.59 ( 36.6$)$ \\
\hline GlcNAc & $3.49 \quad(68.5)$ & $2.48 \quad(57.5)$ \\
\hline GalNAc & & 0.17 ( 3.9$)$ \\
\hline Total & $6.74 \quad(147.8)$ & $7.58 \quad(176.6)$ \\
\hline
\end{tabular}

far from that of the standard globular proteins.

\section{Carbohydrate analysis}

Although the total content of hexoses was reported previously, ${ }^{4,24)}$ no detailed studies have been done on the composition and distribution of carbohydrates in bovine SIgA. Therefore, using gas-liquid chromatography, the composition of neutral and amino hexoses was determined in the present study. The results are summarized in Table I. The bovine colostral SIgA was composed of $6.74 \%$ neutral and amino hexoses, consisting of fucose, mannose, galactose and glucosamine in the amounts shown in Table I. Using the range of data for human $\mathrm{SIgA}^{11)}$ as a basis for comparison, significant differences were found in the contents of both neutral and amino hexoses. The amount of fucose in bovine SIgA is only about a half of that in human SIgA. Less amounts of mannose and galactose (about $70 \%$ ) are also found in bovine SIgA. On the other hand, the amount of glucosamine in bovine $\mathrm{SIgA}$ is $40 \%$ higher than that in human SIgA.

No galactosamine was detected in the present study, though it has been reported to constitute $0.8 \%$ of bovine FSC. ${ }^{25)}$ It was also found in human $\operatorname{SIgA},{ }^{11)}$ but was distributed in the $\mathrm{H}$ chain rather than in $\mathrm{SC}$, and was reported in human FSC only in trace amounts. $^{26)}$

\section{REFERENCES}

1) J. Mestecky, R. Kulhavy and F. W. Kraus, J. Immunology, 108, 738 (1972).

2) M. S. Halpern and M. E. Koshland, J. Immunology, 111, 1653 (1973).

3) R. Komar, E. C. Abson and T. K. S. Mukkur, Immunochemistry, 12, 323 (1975).

4) J. E. Butler, Biochim. Biophys. Acta, 251, 435 (1971).

5) J. E. Butler, L. Peterson and P. L. McGivern, Mol. Immunology, 17, 757 (1980).

6) Y. Kanamaru, R. Niki and S. Arima, Agric. Biol. Chem., 41, 1203 (1977).

7) K. Weber and M. Osborn, J. Biol. Chem., 244, 4406 (1969).

8) R. M. Zacharius and T. E. Zell, Anal. Biochem., 30, 148 (1969).

9) M. E. Groves and W. G. Gordon, Biochim. Biophys. Acta, 194, 421 (1969).

10) T. B. Tomasi and J. Bienenstock, "Advances in Immunology," Vol. 9, ed. by F. Dixon and H. Kunkel, Academic Press Inc., New York, N. Y., 1978 , p. 1

11) M. Tomana, J. Mestecky and W. Niedermeier, $J$. Immunology, 108, 1631 (1972).

12) W. Niedermeier, Anal. Biochem., 40, 465 (1971).

13) J. P. Mach and J. J. Pahud, J. Immunology, 106, 552 (1971).

14) G. Virella and R. M. E. Parkhouse, Immunochemistry, 10, 213 (1973).

15) K. L. Knight, M. L. Vetter and T. R. Malek, $J$. Immunology, 115, 595 (1975).

16) A. P. Johnstone and L. E. Mole, Biochem. J., 167, 245 (1977).

17) T. B. Tomasi and H. M. Grey, Progr. Allergy, 16, 81 (1972).

18) H. M. Grey, C. A. Abel, W. J. Yount and H. G. Kunkel, J. Exp. Med., 128, 1223 (1968).

19) Y. Kanamaru, T. Fujita, S. Ohya, H. Usui, Y. Kuzuya and T. Tanahashi, Res. Bull. Fac. Agr. Gifu Univ., 43, 215 (1980).

20) M. S. Halpern and M. E. Koshland, Nature, 228, 1276 (1970).

21) J. Mestecky, J. Zikan and W. T. Butler, Science, 171, 1163 (1971).

22) K. Kobayashi, J. P. Vaerman, H. Bazin, A. M. Lebacq-Verheyden and J. F. Heremans, $J$. Immunology, 111, 1590 (1973).

23) R. Komar and T. K. S. Mukkur, Can. J. Biochem. 53, 943 (1975).

24) J. P. Mach, J. J. Pahud and H. Isliker, Nature, 223, 952 (1969).

25) R. S. Labib, N. J. Calvanico and T. B. Tomasi, J. Biol. Chem., 251, 1969 (1976).

26) K. Sletten, T. B. Christensen and P. Brandtzaeg, Immunochemistry, 12, 783 (1975).

27) Y. Kanamaru, Y. Kuzuya and T. Tanahashi, Agric. Biol. Chem., 46, 1531 (1982). 